\title{
EPIDEMIOLOGÍA DEL SÍNDROME DE GUILLAIN-BARRÉ EN EL PERÚ
}

\author{
César V. Munayco ${ }^{1, a, b}$, Mirtha G. Soto Cabezas ${ }^{1, a}$, Mary F. Reyes ${ }^{1, a, c}$, Johans A. Arica Gutiérrez ${ }^{1, a, d}$, \\ Omar Napanga Saldaña ${ }^{2, a, e}$
}

\begin{abstract}
RESUMEN
Objetivos. Describir el comportamiento epidemiológico del síndrome de Guillain-Barré (SGB) en el Perú. Materiales y métodos. Estudio descriptivo de datos secundarios sobre egresos hospitalarios del Ministerio de Salud (MINSA) durante el periodo 2012 - 2017, egresos hospitalarios de EsSalud y de SuSalud entre 2015 y 2017, y datos nacionales de mortalidad entre 2014-2016. La identificación del SGB en las bases de datos se realizó utilizando la Clasificación Internacional de Enfermedades, $10^{\mathrm{a}}$ versión, mediante el código G610. Se estimó la incidencia, tasa de letalidad y mortalidad por grupos de edad, sexo y departamento. Resultados. En la base de datos de egresos hospitalarios del MINSA se identificaron un total de 955 casos de SBG entre 2012 y 2017, con una mayor frecuencia en hombres y en la población de 20 a 59 años. La incidencia nacional de SGB por cada 100 mil habitantes fue de 0,62 (2015), 0,92 (2016) y 0,91 (2017), siendo más alta en adultos mayores y en hombres. La tasa de letalidad global fue de $3,5 \%$, y fue más alta en los mayores de 60 años. El promedio del tiempo de hospitalización fue de $16 \pm 22$ días.. Además, Lima es el departamento que concentró más del $40 \%$ de casos de SGB y Cusco, Lima, Callao, Lambayeque y Arequipa tuvieron la mayor incidencia. Conclusiones. Este estudio proporciona información relevante para entender mejor el comportamiento epidemiológico del SGB en el Perú, y así poder garantizar una atención adecuada de los pacientes a nivel nacional.
\end{abstract}

Palabras clave: Síndrome de Guillain-Barré; Epidemiología; Perú (fuente: DeCS BIREME).

\section{EPIDEMIOLOGY OF GUILLAIN-BARRÉ SYNDROME IN PERU}

\begin{abstract}
Objective. To describe the epidemiological behavior of Guillain-Barré syndrome (GBS) in Peru. Materials and Methods. Descriptive study of secondary data on the Ministry of Health (MINSA) hospital discharges during the period 2012-2017; EsSalud and SuSalud hospital discharges between 2015 and 2017; and national mortality data between 2014-2016. The identification of GBS in the databases was carried out using the International Classification of Diseases, 10th version, by means of code G610. Incidence, fatality rate, and mortality were estimated by age, sex, and department group. Results. A total of 955 cases of GBS were identified in the database of MINSA hospital discharges between 2012 and 2017, with a higher frequency in men, within the 20 to 59-year old population. The national incidence of GBS per 100,000 inhabitants was 0.62 (2015), 0.92 (2016), and 0.91 (2017), being higher in older adults and in men. The overall case fatality rate was $3.5 \%$, and was highest in those over 60 . The average length of hospitalization was $16 \pm 22$ days. In addition, Lima is the department that accounted for more than $40 \%$ of all GBS cases; and Cusco, Lima, Callao, Lambayeque, and Arequipa had the highest incidence. Conclusions. This study provides relevant information to better understand the epidemiological behavior of GBS in Peru, and thus ensure adequate patient care nationally.
\end{abstract}

Keywords: Guillain-Barré syndrome; epidemiology; Peru (source: MeSH NLM).

Grupo de Trabajo de Investigación Epidemiológica y Evaluación de Intervenciones Sanitarias. Centro Nacional de Epidemiología, Prevención y Control de Enfermedades. Lima, Perú.

2 Dirección de Inteligencia Sanitaria. Centro Nacional de Epidemiología, Prevención y Control de Enfermedades. Lima, Perú.

a Médico cirujano; ${ }^{\mathrm{b}}$ doctor en Salud Pública; ${ }^{\mathrm{c}}$ magíster en Epidemiología; ${ }^{\mathrm{d}}$ magíster en Sistemas de información Geográfica Aplicada; ${ }^{\mathrm{e}}$ especialista en Epidemiología de Campo.

Recibido: 12/06/2018 Aprobado: 16/01/2019 En línea: 20/03/2019

Citar como: Munayco CV, Soto MG, Reyes MF, Arica JA, Napanga O. Epidemiología del síndrome de Guillain-Barré en el Perú. Rev Peru Med Exp Salud Publica. 2019;36(1):10-6. doi:10.17843/rpmesp.2019.361.3729. 


\section{INTRODUCCIÓN}

El síndrome de Guillain-Barré (SGB) es un conjunto de neuropatías caracterizado por debilidad motora progresiva, disminución y ausencia de reflejos profundos ${ }^{(1)}$. Es una enfermedad autoinmune y desencadenada en dos tercios de los casos, por una infección precedente, con mayor frecuencia infecciones respiratorias o gastrointestinales ${ }^{(2)}$.

La incidencia SGB ha sido estimada en países como Estados Unidos y Europa. En un estudio publicado por Amer et al. ${ }^{(3)}$, se estimó que la incidencia anual de SGB entre 2000 y 2004 fue entre 1,65 a 1,79 por 100000 habitantes. Además, un meta-análisis estimó que la tasa de incidencia de SGB para todos los grupos de edad estudiados fue de 0,62 a 2,66 por 100000 personas-año, y que la incidencia se incrementó en edades por encima de 80 años ${ }^{(4)}$.

En Perú, la información sobre la epidemiología del SGB es limitada. Así, una serie de casos publicada por Ballón-Manrique et al., señala que, los casos de SGB se presentan principalmente en adultos de 20 a 60 años, con igual frecuencia entre hombres y mujeres, y que el subtipo axonal es el más frecuente ${ }^{(5)}$. Suarez et al., estudiaron un brote de parálisis flácida aguda en adultos relacionado con echovirus, en el departamento de Ancash. En este brote se identificaron 16 casos de parálisis flácida aguda, los que en su mayoría fueron hombres con una edad media de 41 años, y con antecedentes de infección respiratoria (6). Además, la tasa de letalidad, en este brote fue de $8,3 \%$.

Actualmente, no se cuenta con información sobre el comportamiento epidemiológico de este síndrome, ni se ha estimado su incidencia, letalidad o tasa de mortalidad en el Perú. Asimismo, en mayo de 2018 se emitió una alerta epidemiológica por un número importante de nuevos casos de SGB en el departamento de La Libertad (más de 20 casos), y se recibieron notificaciones de otros departamentos ${ }^{(7)}$. Por ello, el objetivo del presente estudio fue describir el comportamiento epidemiológico del SGB en el Perú, utilizando las bases de datos de diversas instituciones. Además, se estimó el número de egresos hospitalarios de SGB a nivel nacional para el 2018.

\section{MATERIALES Y MÉTODOS}

Se realizó un estudio descriptivo de datos secundarios sobre egresos hospitalarios del Ministerio de Salud (MINSA) durante el periodo 2012 y 2017 , egresos hospitalarios de la Seguridad Social (EsSalud) y la Superintendencia Nacional de Salud (SuSalud) entre 2015 y 2017, y del Registro Civil o Sistema de Registro de Hechos Vitales entre 2014-2016 del MINSA (base de datos de mortalidad).

La base de datos nacional de egresos hospitalarios del MINSA recoge datos de la epicrisis contenida en la Historia

\section{MENSAJES CLAVE}

Motivación para realizar el estudio. El incremento de casos de síndrome de Guillain-Barré durante mayo del 2018 generó la necesidad de contar con información epidemiológica sobre este problema de salud. Lamentablemente, la información sobre esta patología es limitada en el país. Se realizó un análisis de todas las fuentes que tuvieran datos sobre este síndrome.

Principales hallazgos. El SGB fue más frecuente en hombres y el grupo de edad más afectado fue el de 20 a 59 años. La tasa de letalidad global fue de 3,5\% y fue más alta en los mayores de 60 años. El promedio de días de hospitalización fue de $16 \pm 22$ días.

Implicancias. Este estudio contribuye al conocimiento epidemiológico del síndrome de Guillain-Barré en el Perú.

Clínica del paciente, en algunos hospitales que cuentan con la plataforma SIS-GalenPlus, la usan como fuente de datos para los egresos hospitalarios ${ }^{(8,9)}$. Estos datos son enviados por los hospitales y centros de salud con servicios de hospitalización. La base de datos contiene información de edad, sexo, residencia habitual del paciente, fechas de ingreso y egreso (estancia hospitalaria) y condición de egreso.

Los datos de los egresos hospitalarios de SGB de las Sanidades de FuerzasArmadasy Policialesyestablecimientos de salud privados fueron obtenidos del reporte de SuSalud, ya que no usan el sistema de egresos hospitalarios del MINSA. Los egresos hospitalarios de los casos del SGB de EsSalud fueron obtenidos del Sistema de Gestión Hospitalaria, que es un sistema que registra los datos de todos los pacientes ambulatorios y hospitalizados en EsSalud.

La base de datos del Registro Civil o sistema de registro de hechos vitales recoge datos de los certificados de defunción que son llenados por el personal de salud a nivel nacional ${ }^{(10)}$. Esta base de datos fue utilizada para calcular la tasa de mortalidad por SGB.

La identificación de SGB en las bases de datos estudiadas se realizó utilizando el código G610 de la Clasificación Internacional de Enfermedades, $10^{\text {a }}$ versión (CIE-10). Cabe resaltar que, no se ha realizado ninguna validación de los diagnósticos de estas bases de datos.

Se realizó un análisis descriptivo de las características de los pacientes con SGB a través de tablas de frecuencias relativas y absolutas para las variables categóricas (sexo, condición de egreso), y media y desviación estándar para variables numéricas (edad, estancia hospitalaria). Además, se calculó la tasa de incidencia por edad, sexo y por departamentos, y la tasa de letalidad por edad. La tasa de mortalidad se estimó por edad y sexo para los años 2014, 2015 y 2016. Para el cálculo de las tasas se 
utilizó la población estimada por el Instituto Nacional de Estadística e Informática (INEI) disponibles en https:// www.inei.gob.pe/estadisticas/indice-tematico/poblacion-yvivienda/. Finalmente, se diseñaron mapas para mostrar la distribución geográfica de los casos de SGB por departamento y por años.

Con la finalidad de tener una línea de base para el 2018, se estimaron los egresos hospitalarios mensuales para todo el país usando los datos mensuales entre 2015 y 2017, a través del modelo de espacio-estado ${ }^{(11)}$, para lo cual se usó el comando «stlf» del paquete «forecast» en R. Este «comando stlf» es un acrónimo del método "Seasonal and Trend decomposition using Loess», que es un método robusto para descomponer series de tiempo que usa el método Loess para estimar relaciones no lineales ${ }^{(12)}$. Este método permitió ajustar los datos al modelo espacio-estado o variables latentes y estimar los casos para el 2018.

Con relación a los aspectos éticos, para garantizar la confidencialidad de la información se obtuvieron las bases de datos, omitiéndose los nombres y datos de identificación de las personas, además se contó con la autorización institucional para la difusión de los datos.

\section{RESULTADOS}

En la base de datos de egresos hospitalarios del MINSA se identificaron un total de 955 registros de casos de SBG entre 2012 y 2017.

El $50 \%$ de los casos de SGB tuvieron una edad menor a 38 años. El mayor porcentaje de los casos se reportó en el grupo de 20 a 59 años y el $60,6 \%$ de casos fueron hombres. La mediana del tiempo de hospitalización fue de 11 días, mientras que el $64 \%$ de los casos de SGB tuvieron un tiempo de incidencia menor a 15 días. El tiempo de hospitalización varió con la edad: en los menores de 20 años, la mitad de los casos tuvo un tiempo de hospitalización de diez días, en el grupo de 20 a 59 años fue de 11 días y en
Tabla 1. Características generales de los egresos hospitalarios de los casos síndrome de Guillain-Barré de los establecimientos de salud del Ministerio de Salud, Perú 2012-2017.

\begin{tabular}{lcc}
\hline $\begin{array}{l}\text { Características de los pacientes } \\
\text { con SGB }\end{array}$ & N=955 & Porcentaje \\
\hline $\begin{array}{l}\text { Grupos de edad (años) } \\
\quad \text { Mediana (RI) }\end{array}$ & $40(19-56)$ & - \\
\hline $0-9$ & 134 & 14,0 \\
\hline $10-19$ & 112 & 11,8 \\
\hline $20-59$ & 531 & 55,6 \\
\hline 60 o más & 178 & 18,6 \\
\hline Sexo & & \\
\hline Masculino & 579 & 60,6 \\
\hline Femenino & 376 & 39,4 \\
\hline Tiempo de hospitalización (días) & & \\
\hline Mediana (RI) & $11(6-19)$ & - \\
\hline$<15$ & 614 & 64,3 \\
\hline 15 a 29 & 225 & 23,6 \\
\hline 30 a 60 & 83 & 8,7 \\
\hline$>60$ & 33 & 3,5 \\
\hline Condición de egreso & & \\
\hline Alta & 922 & 96,5 \\
\hline Fallecido & 33 & 3,5 \\
\hline Tasa de letalidad por grupo etario (años) & & \\
\hline $0-9$ & $1 / 134$ & 0,7 \\
\hline $10-19$ & $3 / 112$ & 2,7 \\
\hline $20-59$ & $14 / 531$ & 2,6 \\
\hline$>60$ & $15 / 178$ & 8,4 \\
\hline
\end{tabular}

SGB: síndrome de Guillain-Barré; RI: rango intercuartil (p25 y p75)

Fuentes de datos: Base de egresos hospitalarios del Ministerio de Salud

los mayores de 60 años fue de 13 días. La tasa de letalidad para todas las edades fue de $3,5 \%$, pero en los mayores de 60 años, fue la más alta $(8,4 \%)$ (Tabla 1$)$.

En la Tabla 2, se muestra el número de casos y el número de fallecidos de SGB, además, de las tasas de incidencia y

Tabla 2. Casos, incidencia y mortalidad de síndrome de Guillain-Barré por grupos de edad y sexo, Perú 2014-2017

\begin{tabular}{|c|c|c|c|c|c|c|c|c|c|c|c|c|}
\hline \multirow{2}{*}{$\begin{array}{l}\text { Características de la } \\
\text { población }\end{array}$} & \multicolumn{3}{|c|}{ Número de casos } & \multicolumn{3}{|c|}{$\begin{array}{l}\text { Incidencia por } 100 \\
\text { mil habitantes }\end{array}$} & \multicolumn{3}{|c|}{ Número de fallecidos } & \multicolumn{3}{|c|}{$\begin{array}{l}\text { Mortalidad por } 100 \\
\text { mil habitantes }\end{array}$} \\
\hline & 2015 & 2016 & 2017 & 2015 & 2016 & 2017 & 2014 & 2015 & 2016 & 2014 & 2015 & 2016 \\
\hline \multicolumn{13}{|l|}{ Grupos de edad (años) } \\
\hline 0 a 9 & 16 & 30 & 27 & 0,28 & 0,52 & 0,47 & 0 & 0 & 3 & 0,00 & 0,00 & 0,05 \\
\hline 10 a 19 & 30 & 29 & 30 & 0,52 & 0,50 & 0,52 & 0 & 0 & 5 & 0,00 & 0,00 & 0,09 \\
\hline 20 a 59 & 99 & 171 & 162 & 0,60 & 1,02 & 0,95 & 3 & 25 & 5 & 0,02 & 0,15 & 0,03 \\
\hline 60 o más & 69 & 61 & 71 & 2,29 & 1,96 & 2,20 & 6 & 9 & 13 & 0,20 & 0,30 & 0,42 \\
\hline \multicolumn{13}{|l|}{ Sexo } \\
\hline Masculino & 137 & 186 & 172 & 0,88 & 1,18 & 1,08 & 9 & 11 & 16 & 0,06 & 0,07 & 0,10 \\
\hline Femenino & 77 & 105 & 118 & 0,50 & 0,67 & 0,74 & 0 & 23 & 10 & 0,00 & 0,15 & 0,06 \\
\hline Total nacional & 214 & 291 & 290 & 0,69 & 0,92 & 0,91 & 9 & 34 & 26 & 0,03 & 0,11 & 0,08 \\
\hline
\end{tabular}

Fuentes de datos: Base egresos hospitalarios del Ministerio de Salud; Reporte del Sistema de Gestión Hospitalaria de EsSalud; Base de Registros de datos de SuSalud; Sistemas de registro de hechos vitales (mortalidad). Para el cálculo de las tasas se utilizó la población estimada por el Instituto Nacional de Estadística e Informática. 
mortalidad, por grupos de edad y sexo. Durante los años 2015, 2016 y 2017, se registraron 214, 291 y 290 egresos hospitalarios de SGB, respectivamente. La incidencia nacional por 100 mil habitantes para estos mismos años fue de 0,62, 0,92 y 0,91, respectivamente. Según los grupos de edad, los mayores de 60 años obtuvieron la incidencia más alta, y la incidencia fue mayor en los hombres en comparación con las mujeres, en todos los años estudiados.

A nivel nacional, utilizando los registros del sistema de hechos vitales entre 2014 y 2016, se estimó un total de 69 fallecidos por SGB, siendo las tasas de mortalidad estimadas de 0,03, 0,11 y 0,08 por 100 mil habitantes, y la tasa de mortalidad fue más alta en los mayores de 60 años (Tabla 2).

Los departamentos que registraron mayor número de egresos hospitalarios (2015-2017) por SGB fueron: Lima, Arequipa, Cusco, La Libertad y Lambayeque (Tabla 3). El departamento de Lima concentró más del $40 \%$ de los casos de SGB en los años estudiados. Por otro lado, los departamentos con mayor incidencia fueron: Cusco, Lima, Callao, Lambayeque y Arequipa.

En la Figura $1 \mathrm{~A}$, se muestra la tendencia de los egresos hospitalarios por SGB y la distribución espacial por departamentos para los años 2015, 2016 y 2017 (Figura 1B-D). La tendencia de los egresos de SBG es estable en el tiempo, con algunos picos durante los primeros meses del año durante el periodo 2015 y 2017 . Además, en promedio se registraron 22 egresos hospitalarios mensuales de SGB a nivel nacional. La Figura $1 \mathrm{~A}$, muestra los egresos hospitalarios estimados por mes para el 2018, basado en lo reportado durante los tres años anteriores.

El departamento de Lima siempre presentó el mayor número de casos en los tres años estudiados (Figura 1BD). En 2017, además de Lima, los departamentos de La Libertad, Cusco, Arequipa y la región Callao presentaron más de 15 casos por año (Figura 1D).

Tabla 3. Número de egresos hospitalarios e incidencia de síndrome de Guillain-Barré por departamentos, Perú 2015-2017

\begin{tabular}{|c|c|c|c|c|c|c|}
\hline \multirow{2}{*}{ Departamento } & \multicolumn{3}{|c|}{ Número de egresos hospitalarios por SGB } & \multicolumn{3}{|c|}{ Incidencia por 100 mil habitantes } \\
\hline & 2015 & 2016 & 2017 & 2015 & 2016 & 2017 \\
\hline Amazonas & 0 & 1 & 0 & 0,00 & 0,24 & 0,00 \\
\hline Ancash & 8 & 9 & 11 & 0,70 & 0,78 & 0,95 \\
\hline Apurímac & 2 & 2 & 0 & 0,44 & 0,43 & 0,00 \\
\hline Arequipa & 13 & 14 & 20 & 1,01 & 1,08 & 1,52 \\
\hline Ayacucho & 2 & 3 & 4 & 0,29 & 0,43 & 0,57 \\
\hline Cajamarca & 9 & 9 & 11 & 0,59 & 0,59 & 0,72 \\
\hline Callao & 6 & 16 & 16 & 0,59 & 1,56 & 1,54 \\
\hline Cusco & 15 & 14 & 20 & 1,14 & 1,06 & 1,50 \\
\hline Huancavelica & 3 & 3 & 1 & 0,61 & 0,60 & 0,20 \\
\hline Huánuco & 3 & 7 & 3 & 0,35 & 0,81 & 0,34 \\
\hline Ica & 4 & 7 & 6 & 0,51 & 0,88 & 0,75 \\
\hline Junín & 5 & 13 & 9 & 0,37 & 0,96 & 0,66 \\
\hline La Libertad & 15 & 15 & 19 & 0,81 & 0,80 & 1,00 \\
\hline Lambayeque & 12 & 14 & 13 & 0,95 & 1,10 & 1,02 \\
\hline Lima & 95 & 142 & 127 & 0,97 & 1,42 & 1,25 \\
\hline Loreto & 5 & 5 & 2 & 0,48 & 0,48 & 0,19 \\
\hline Madre De Dios & 1 & 0 & 2 & 0,73 & 0,00 & 1,39 \\
\hline Moquegua & 0 & 0 & 1 & 0,00 & 0,00 & 0,54 \\
\hline Pasco & 2 & 1 & 4 & 0,66 & 0,33 & 1,30 \\
\hline Piura & 4 & 3 & 4 & 0,22 & 0,16 & 0,21 \\
\hline Puno & 1 & 3 & 3 & 0,07 & 0,21 & 0,21 \\
\hline San Martin & 4 & 7 & 8 & 0,48 & 0,82 & 0,93 \\
\hline Tacna & 3 & 0 & 5 & 0,88 & 0,00 & 1,43 \\
\hline Tumbes & 2 & 1 & 0 & 0,84 & 0,42 & 0,00 \\
\hline Ucayali & 0 & 2 & 1 & 0,00 & 0,40 & 0,20 \\
\hline Total nacional & 214 & 291 & 290 & 0,69 & 0,92 & 0,91 \\
\hline
\end{tabular}

SGB: síndrome de Guillain-Barré.

Fuentes de datos: Base egresos hospitalarios MINSA; Reporte del Sistema de Gestión Hospitalaria de EsSalud; Base de Registros de datos SuSalud. Para el cálculo de las tasas se utilizó la población estimada por el Instituto Nacional de Estadística e Informática. 

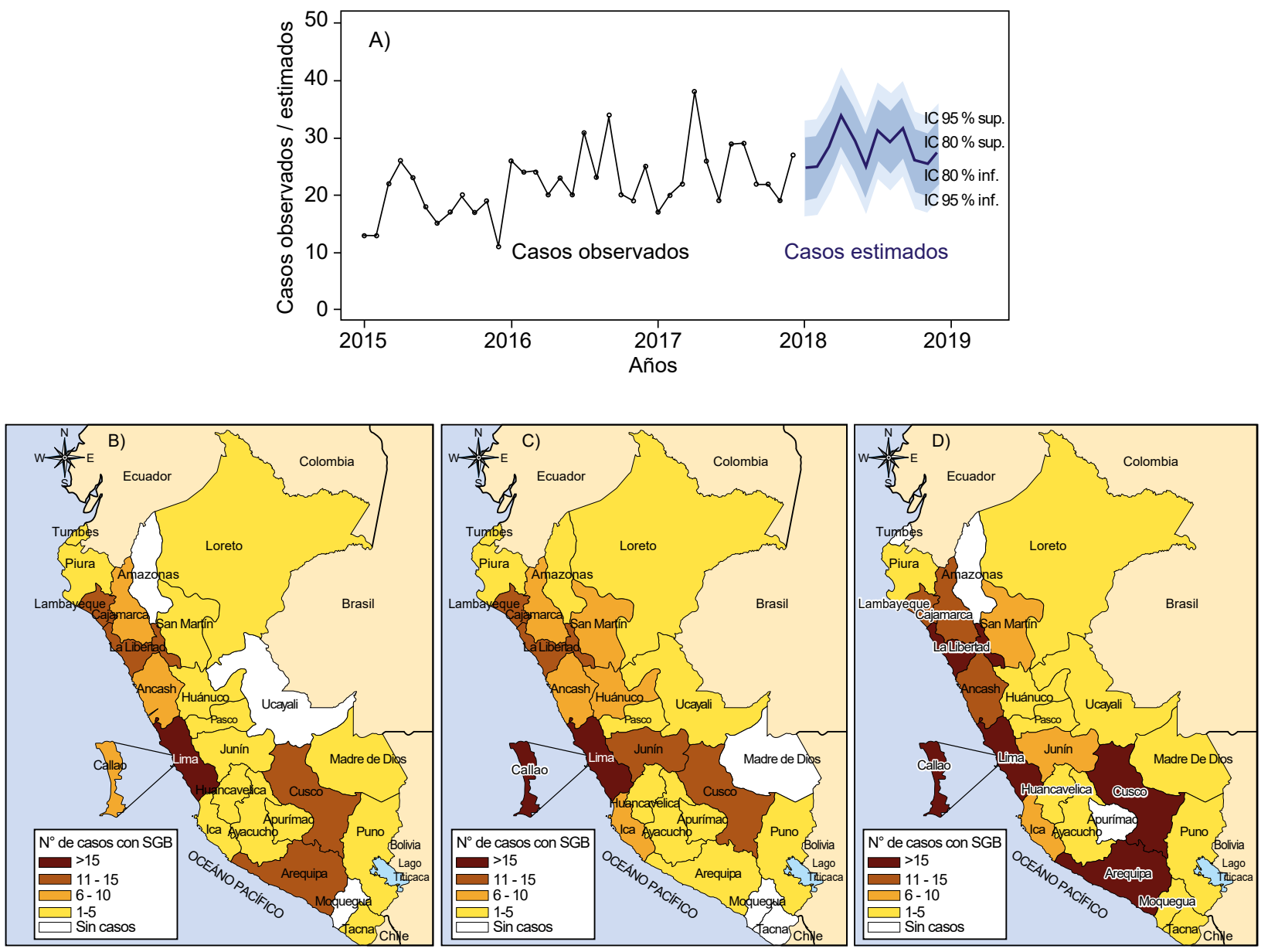

Figura 1. A. Estimación de los egresos hospitalarios de los casos de síndrome de Guillain-Barré de 2018. B. Distribución espacial de los casos de síndrome de Guillain-Barré de 2015. C. Distribución espacial de los casos de síndrome de Guillain-Barré de 2016 . D. Distribución espacial de los casos de síndrome de Guillain-Barré de 2017.

\section{DISCUSIÓN}

El presente estudio muestra que, el SGB fue más frecuente en hombres que en mujeres y el grupo de edad más afectado fue el de 20 a 59 años. Asimismo, la tasa de letalidad global fue de $3,5 \%$, la cual fue más alta en los mayores de 60 años. Por otro lado, la tasa de incidencia más alta se presentó en los mayores de 60 años y en los hombres. Lima es el departamento que concentra más del $40 \%$ de casos de SGB. Los departamentos con mayor incidencia fueron: Cusco, Lima, Callao, Lambayeque y Arequipa.

Los pocos estudios realizados en nuestro país señalan que, los casos de SGB son más frecuentes en hombres y que el promedio de edad varía entre 32 y 44 años ${ }^{(14,5)}$. Estudios realizados en otros países ${ }^{(15-17)}$, encontraron resultados similares a los reportados en el Perú. En el estudio de Olivé et al., realizado en niños menores de 15 años, el SGB fue más frecuente en hombres con una razón de hombre/ mujer de 1,36. En otro estudio realizado por Mao et al. (15), el promedio de edad fue de 31 años, y el porcentaje de hombres y mujeres fue similar. Hallazgos similares fueron encontrados en nuestro estudio, donde el SGB fue más frecuente en hombres y el promedio de edad de los casos por SGB fue de $38 \pm 22$ años.

Apaza et al. ${ }^{(14)}$ encontraron que el tiempo de hospitalización de loscasos deSBG, varíadeacuerdoconeltipoelectrofisiológico, por ejemplo, la polineuropatía desmielinizante inflamatoria aguda y el síndrome de Miller-Fisher tuvieron un tiempo de hospitalización promedio de 21 días, mientras que el tiempo de hospitalización de la neuropatía axonal motora aguda fue de 16 días. Desafortunadamente, en este estudio no se logró identificar los tipos fisiológicos del SGB porque en las bases de datos revisadas no está incluida esa variable, pero se estimó que el tiempo promedio de hospitalización fue de $16 \pm 22$ días y que, a mayor edad, mayor fue el tiempo de hospitalización, es así como los mayores de 60 años tuvieron un tiempo promedio más alto ( $20 \pm 24$ días). 
De acuerdo con el estudio de Olivé et al. ${ }^{(17)}$ realizado en Latinoamérica en pacientes menores de 15 años, la tasa de letalidad por SGB fue de 7\%. En el estudio de Mao et al. ${ }^{(15)}$ realizado en todos los grupos de edad, se encontró una tasa de letalidad de 6\%. Según los hallazgos de este estudio, se encontró una letalidad global más baja de 3,5\%. Además, la letalidad en niños menores de diez años $(0,7 \%)$ fue mucho más baja que la reportado por Olivé et al. (17). Es importante resaltar que, en nuestro estudio la tasa de letalidad en los mayores de 60 años fue de 8,4\%. Estas diferencias en las tasas de letalidad se podrían explicar por el tipo de SGB incluido en cada estudio, como lo muestra el estudio de Mao et al., donde se observa que existen diferencias importantes en la letalidad de acuerdo con el tipo de SGB ${ }^{(15)}$. En este estudio no se logró diferenciar el tipo de SGB, porque ese dato no estuvo disponible en la base de datos revisada.

La incidencia de SGB varía de acuerdo con los grupos de edad y el país. En Finlandia la incidencia de SBG en niños menores de 15 años fue de 0,38 por 100000 habitantes ${ }^{(18)}$, en Curacao la incidencia fue de 2,53 por 100000 habitantes ${ }^{(19)}$. En Estados Unidos la incidencia ajustada varió de 1,65 a 1,79 por 100000 habitantes ${ }^{(20)}$. En nuestro estudio, la incidencia global fluctuó entre 0,69 a 0,91 en los tres años estudiados. Además, la incidencia de SGB fue más alta en hombres que en mujeres y en los mayores de 60 años. Este incremento de la incidencia en los mayores de 60 años, también fue observada en otros estudios ${ }^{(16,19,20)}$. Se estimó la tasa de mortalidad por SGB, no encontrándose estudio para comparar.

Los resultados del estudio muestran una variabilidad geográfica de la incidencia de SGB, donde las tasas más altas se presentaron en Cusco, Lima, Callao, Lambayeque y Arequipa. Los departamentos de la sierra y selva presentaron incidencias más bajas que el promedio nacional. Estas diferencias pueden explicarse por un mayor número de especialistas y mejor infraestructura de los hospitales para hacer el diagnóstico de SGB.

Estudios previos han propuesto que el SGB presenta estacionalidad, aunque no se ha demostrado que exista un significativo incremento de los casos de SGB en ciertos periodos del año ${ }^{(2)}$. En algunos estudios se ha reportado mayor número de casos durante el invierno ${ }^{(21,22)}$, otros en primavera y verano ${ }^{(23)}$. En nuestro estudio no se observa estacionalidad, aunque el número de casos es mayor durante los primeros meses del año. Además, no se dispone de datos suficientes para evaluarla.

Otro punto importante, de este estudio fue estimar los egresos hospitalarios del SGB para que el Centro Nacional de Epidemiología, Prevención y Control de Enfermedades (CDC Perú), tenga los casos esperados para el 2018 y poder compararlos con los casos observados en este año y así definir si se está frente a un incremento por encima de lo esperado de casos de SGB (brote de SGB) en la zona norte del país.

Este estudio presenta algunas limitaciones considerando que los datos utilizados provienen de fuentes secundarias. Entre estas limitaciones, tenemos la calidad de los datos y el sub-registro de los egresos hospitalarios y los datos de mortalidad. Para mejorar la calidad de los mismos se cruzaron los datos de todas las instituciones completando los datos o casos faltantes, o la información inconsistente. El subregistro de los egresos hospitalarios no se pudo determinar, pero se estima que es reducido, debido a la utilización de las epicrisis (ningún paciente puede salir de alta sin epicrisis) y la plataforma SIS-GalenPlus. Además, los responsables de ingresar la información al sistema de egresos hospitalarios son las oficinas de estadística de los hospitales que garantizan el ingreso de estos datos. Por otro lado, se conoce que el subregistro de la mortalidad de los hechos vitales es alrededor del 43,3\% para el año $2015^{(13)}$, y las estimaciones de las tasas de mortalidad incluyeron el subregistro de las muertes por SGB ${ }^{(10)}$. Otra limitación a tomar en cuenta, es que los datos obtenidos corresponden a diferentes periodos de tiempo, lo que no permite comparar adecuadamente los datos a través del tiempo, pero a pesar de ello se puede tener una idea de la evaluación temporal del SGB. Finalmente, es importante destacar que la oferta del diagnóstico y del tratamiento, pueden haber afectado la distribución por departamentos, principalmente en las áreas de sierra y selva del país.

Este estudio, proporciona información epidemiológica importante para entender mejor el comportamiento del SGB en el Perú a través de los egresos hospitalarios, para la planificación de recursos tanto humano como de insumos médicos, garantizando una respuesta adecuada frente a los casos de SGB que se presenten a nivel nacional. Sumado a ello, proporciona la línea basal de casos de SGB para este año y así poder identificar cualquier variación por encima de lo esperado, para garantizar una rápida respuesta.

Agradecimientos: Los autores agradecen a la licenciada Yovanna Seclén por haber compartido la información consolidada de egresos hospitalarios de EsSalud. Además, agradecer a la Sra. María Berto y Dr. José Medina, por haber preparado las consultas de las bases de datos de egresos hospitalarios y mortalidad. Finalmente, queremos agradecer la Superintendencia Nacional de Salud (SuSalud) por haber compartido su reporte de egresos hospitalarios.

Fuentes de financiamiento: autofinanciado.

Conflictos de interés: ninguno que declarar.

Renuncia de responsabilidad: todas las opiniones expresadas en este manuscrito son responsabilidad de los autores y no del Centro Nacional de Epidemiología, Prevención y Control de Enfermedades. 


\section{REFERENCIAS BIBLIOGRÁFICAS}

1. Walling AD, Dickson G. GuillainBarré syndrome. Am Fam Physician. 2013;87(3):191-7.

2. McGrogan A, Madle GC, Seaman HE, de Vries CS. The epidemiology of Guillain-Barré syndrome worldwide. A systematic literature review. Neuroepidemiology. 2009;32(2):150-63. doi: 10.1159/000184748.

3. Alshekhlee A, Hussain Z, Sultan B, Katirji B. Guillain-Barré syndrome: incidence and mortality rates in US hospitals. Neurology. 2008;70(18):1608-13. doi: 10.1212/01.wnl.0000310983.38724.d4.

4. Sejvar JJ, Baughman AL, Wise M, Morgan OW. Population incidence of Guillain-Barré syndrome: a systematic review and meta-analysis. Neuroepidemiology. 2011;36(2):123-33. doi: $10.1159 / 000324710$.

5. Ballón-Manrique B, Campos-Ramos N. Características clínicas y paraclínicas del Síndrome de Guillain-Barré en el Hospital Regional Lambayeque. Rev Neuropsiquiatr. 2017;80(1):22-26. doi: https://doi. org/10.20453/rnp.v80i1.3056.

6. Suárez V, Suárez M, Espinoza M, Ramírez G, Guzmán $S$, et al. Brote de parálisis flácida aguda por echovirus en Ancash. Libro de Resúmenes de VII Congreso Peruano de Enfermedades Infecciosas y Tropicales. Lima, 2001.

7. Centro Nacional de Epidemiología, Prevención y Control de Enfermedades. Alerta Epidemiológica. Notificación Inmediata de casos de Síndrome de Guillain-Barré, Parálisis Flácida y otros síndromes neurológicos agudos en el Perú [Internet]. Lima: MINSA; 2018 [citado el 15 de mayo de 2018]. Disponible en: http://www.dge.gob.pe/portal/docs/ alertas/2018/AE006.pdf

8. Ministerio de Salud. Compendio Estadístico de Egresos Hospitalarios. Perú: 2009 - 2010 [Internet]. Lima: MINSA; 2012 [citado el 15 de mayo de 2018]. Disponible en: http://bvs.minsa.gob.pe/ local/minsa/1834.pdf
9. Universidad Nacional Mayor de San Marcos. SIS-GalenPlus* [Internet]. Lima: Facultad de Medicina de la UNMSM; 2018 [citado el 15 de mayo de 2018]. Disponible en: http://gestionensalud. medicina.unmsm.edu.pe/?tool=sisgalenplus.

10. Ministerio de Salud. Mortalidad General en el Perú 2007 - 2011 [Internet]. Lima: MINSA; 2013 [citado el 15 de mayo de 2018]. Disponible en: http:// www.dge.gob.pe/portal/docs/asis/Asis_ mortalidad.pdf

11. Hyndman R. Major changes to the forecast package 2018 [Internet]. [citado el 15 de mayo de 2018]. Disponible en: https://robjhyndman.com/hyndsight/ forecast3/

12 Cleveland RB, Cleveland WS, McRae JE, Terpenning IJ. STL: A seasonal-trend decomposition procedure based on loess. Journal of Official Statistics. 1990;6(1):3-73.

13. Ministerio de Salud. Indicadores de mortalidad 2016 [Internet]. Lima: MINSA; 2017 [citado el 15 de mayo de 2018]. Disponible en: www.dge.gob.pe/ portal/index.php?option $=$ com_conten t\&view $=$ article $\& \mathrm{id}=648$ : indbas 2014 3\&catid $=7 \&$ Itemid $=204$.

14. Apaza EL. Características clínicas y electrofisiológicas del síndrome de Guillain Barré en el Instituto Nacional de Ciencias Neurológicas, 2008-2012. Tesis para obtener el título de especialista en Neurología. Lima: Universidad Nacional Mayor de San Marcos; 2014.

15. Mao Z, Hu X. Clinical characteristics and outcomes of patients with Guillain-Barre and acquired CNS demyelinating overlap syndrome: a cohort study based on a literature review. Neurol Res. 2014;36(12):1106-13.

16. McGrogan A, Madle GC, Seaman HE, de Vries CS. The epidemiology of GuillainBarre syndrome worldwide. A systematic literature review. Neuroepidemiology. 2009;32(2):150-63.
17. Olive JM, Castillo C, Castro RG, de Quadros CA. Epidemiologic study of Guillain-Barre syndrome in children $<15$ years of age in Latin America. J Infect Dis. 1997;175 Suppl 1:S160-4.

18. Rantala $H$, Uhari $M$, Niemela $M$. Occurrence, clinical manifestations, and prognosis of Guillain-Barre syndrome. Arch Dis Child. 1991;66(6):706-8.

19. van Koningsveld R, Rico R, Gerstenbluth I, Schmitz PI, Ang CW, Merkies IS, et al. Gastroenteritis-associated Guillain-Barre syndrome on the Caribbean island Curacao. Neurology. 2001;56(11):1467-72.

20. Alshekhlee A, Hussain Z, Sultan B, Katirji B. Guillain-Barre syndrome: incidence and mortality rates in US hospitals. Neurology. 2008;70(18):1608-13.

21. Paolino E, Govoni V, Tola MR, Casetta I, Granieri E. Incidence of the Guillain-Barre syndrome in Ferrara, northern Italy, 1981-1987. Neuroepidemiology. 1991;10(3):105-11. doi: $10.1159 / 000110254$.

22. Govoni V, Granieri E, Tola MR, Casetta I, Ruppi P, Vaghi L. The frequency of clinical variants of Guillain-Barre syndrome in Ferrara, Italy. J Neurology. 1999;246(11):1010-4.

23. Rocha MS, Brucki SM, Carvalho AA, Lima UW. Epidemiologic features of Guillain-Barre syndrome in Sao Paulo, Brazil. Arq. Neuro-Psiquiatr. 2004;62(1):33-7. doi: 10.1590/S0004282X2004000100006

Correspondencia: César Vladimir Munayco Escate

Centro Nacional de Epidemiologia, Prevención y Control de Enfermedades CDC Perú. Dirección: Calle Saturno 117, Ate, Lima - Perú. Teléfono: +51982508166

Correoelectrónico:cmunayco@dge.gob.pe 